\title{
Some New Comparison Theorems for Double Splittings of Matrices
}

\author{
Cui-Xia Li and Shi-Liang Wu* \\ School of Mathematics and Statistics, Anyang Normal University, Anyang, 455000, P.R. China
}

Received: 22 Sep. 2013, Revised: 20 Dec. 2013, Accepted: 21 Dec. 2013

Published online: 1 Sep. 2014

\begin{abstract}
In this paper, we further investigate the double splitting iterative methods for solving linear systems. Building on the previous work by Song and Song [Convergence for nonnegative double splittings of matrices, Calcolo, (2011) 48: 245-260], some new comparison theorems for the spectral radius of double splittings of matrices under suitable conditions are presented.
\end{abstract}

Keywords: Linear systems, matrix, double splitting, single splitting, spectral radius, comparison theorem.

\section{Introduction}

Consider the following linear system

$A x=b$,

where $A \in \mathbb{R}^{n \times n}$ is a nonsingular matrix, $b \in \mathbb{R}^{n \times 1}$ is a given vector and $x \in \mathbb{R}^{n \times 1}$ is an unknown vector. In order to solve the linear system (1) by iterative methods, the coefficient matrix $A$ is split into

$A=M-N$,

where $M$ is nonsingular, is called a single splitting of $A$ in [1]. Based on the above matrix splitting, the basic iterative method for solving (1) is

$x^{k+1}=M^{-1} N x^{k}+M^{-1} b \equiv T x^{k}+M^{-1} b$,

where $k=0,1, \ldots$ and $T=M^{-1} N$ is the iteration matrix in (2). Obviously, the iterative method (2) converges to the unique solution of the linear system (1) if and only if the spectral radius $\rho\left(M^{-1} N\right)$ of the iteration matrix is smaller than 1 . The spectral radius of the iteration matrix is decisive for the convergence and stability, and the smaller it is, the faster the iterative method converges when the spectral radius is smaller than 1. So far, many comparison theorems of single splitting of matrices have been presented in some papers and books $[2-8,13]$.

In [1], Woźnicki introduced a double splitting of $A$, i.e., splitting the matrix $A$ in the form

$A=P-R-S$, where $P$ is a nonsingular matrix. The corresponding iterative scheme is spanned by three successive iterations,

$x^{k+1}=P^{-1} R x^{k}+P^{-1} S x^{k-1}+P^{-1} b, k=0,1,2, \ldots$

which can be rewritten in the equivalent form

$\left[\begin{array}{c}x^{k+1} \\ x^{k}\end{array}\right]=\left[\begin{array}{cc}P^{-1} R & P^{-1} S \\ I & 0\end{array}\right]\left[\begin{array}{c}x^{k} \\ x^{k-1}\end{array}\right]+\left[\begin{array}{c}P^{-1} b \\ 0\end{array}\right]$,

where $I$ is the identity matrix. The iterative method given by (5) converges to the unique solution of (1) for all initial vectors $x^{0}, x^{1}$ if and only if the spectral radius of the iteration matrix,

$W=\left[\begin{array}{cc}P^{-1} R & P^{-1} S \\ I & 0\end{array}\right]$

is less than one, i.e., $\rho(W)<1$.

Recently, some convergence and comparison results for double splittings of matrices are presented. In [9], Shen and Huang presented some convergence theorems for the double splitting of a monotone matrix or a Hermitian positive definite matrix and obtained two comparison theorems for two double splittings of a monotone matrix. Compared with some results [9], some improved convergence and comparison results for double splitting of a Hermitian positive definite matrix are proposed in [10]. In [12], some convergence results for double splittings of a non-Hermitian positive semidefinite matrix are established. In [11], a comparison theorem for double splittings of different monotone matrices is given. In [13], some convergence and comparison results for

\footnotetext{
*Corresponding author e-mail: wushiliang1999@126.com, slwu@ aynu.edu.cn
} 
nonnegative double splittings of matrices are given. In this paper, building on the previous work [13], our basic purpose here is to derive some new comparison theorems for the spectral radius of double splittings of matrices.

\section{Preliminaries}

For convenience, we give some of the notations, definitions and lemmas which will be used in the sequel.

The matrix $A$ is called nonnegative and denoted $A \geq 0$ if $a_{i j} \geq 0$ for $i, j=1,2, \ldots, n$. We write $A \geq B(A>B)$ if $a_{i j} \geq b_{i j}\left(a_{i j}>b_{i j}\right)$ for $i, j=1,2, \ldots, n$. The matrix $A$ is called to be a monotone matrix if $A^{-1} \geq 0$. Matrix $A$ is an $L$-matrix if $a_{i i}>0(i=1, \ldots, n)$ and $a_{i j}<0$ for all $i, j=1, \ldots, n ; i \neq j$.

Definition 1. $[9,13]$ Let $A$ be a nonsingular matrix. Then the double splitting $A=P-R-S$ is

- convergent if and only if $\rho(W)<1$;

- a regular double splitting if $P^{-1} \geq 0, R \geq 0$ and $S \geq 0$;

- a weak regular double splitting if $P^{-1} \geq 0, P^{-1} R \geq 0$ and $P^{-1} S \geq 0$;

- a nonnegative splitting if $P^{-1} R \geq 0$ and $P^{-1} S \geq 0$.

Definition 2. Let $A$ be a nonsingular matrix. The double splitting $A=P-R-S$ is an $M$-double splitting if $P$ is an $M$-matrix and $R \geq 0$ and $S \geq 0$.

Lemma 1. [3] Let $A \geq 0$. Then

$\alpha x \leq A x, x \geq 0$, implies $\alpha \leq \rho(A)$,

and

$A x \leq \beta x, x>0$, implies $\rho(A) \leq \beta$.

Lemma 2. [9] Let $A^{-1} \geq 0$ and $A=P-R-S$ be a weak regular double splitting. Then $\rho(W)<1$.

Lemma 3. [15] Let $A \in \mathbb{R}^{n \times n}$ and $A=M_{1}-N_{1}=$ $M_{2}-N_{2}$ be $M$-splittings of $A$ (i.e., $M_{i}$ are $M$-matrices, $\left.N_{i} \geq 0, i=1,2\right)$ and

$N_{1} \geq N_{2}, N_{1} \neq N_{2}, N_{2} \neq 0$.

Then exactly one of the following statements holds:

(1) $0 \leq \rho\left(M_{2}^{-1} N_{2}\right)<\rho\left(M_{1}^{-1} N_{1}\right)<1$. In addition, if $A$ is irreducible, the first inequality is also strict.

(2) $\rho\left(M_{2}^{-1} N_{2}\right)=\rho\left(M_{1}^{-1} N_{1}\right)=1$.

(3) $\rho\left(M_{2}^{-1} N_{2}\right)>\rho\left(M_{1}^{-1} N_{1}\right)>1$.

\section{Comparison theorems}

Let

$A=P_{1}-R_{1}-S_{1}=P_{2}-R_{2}-S_{2}$

be two double splittings of $A$. Then we define

$W_{1}=\left[\begin{array}{cc}P_{1}^{-1} R_{1} & P_{1}^{-1} S_{1} \\ I & 0\end{array}\right]$ and $W_{2}=\left[\begin{array}{cc}P_{2}^{-1} R_{2} & P_{2}^{-1} S_{2} \\ I & 0\end{array}\right]$.

In [13], some comparison theorems for the spectral radius of double splittings of monotone matrices are given, which are described as follows.
Theorem 1. [13] Let $A^{-1} \geq 0$, and let the two double splittings (7) be nonnegative and convergent. Suppose

$P_{1} \leq P_{2}, S_{1} \leq S_{2}$,

then

$\rho\left(W_{1}\right) \leq \rho\left(W_{2}\right)$.

Corollary 1. [13] Let $A^{-1} \geq 0$, and let the two double splittings (7) be nonnegative and convergent. Suppose

$R_{1} \leq R_{2}, S_{1} \leq S_{2}$,

then

$\rho\left(W_{1}\right) \leq \rho\left(W_{2}\right)$.

Theorem 2. [13] Let $A^{-1} \geq 0, A=P_{1}-R_{1}-S_{1}$ be regular double splitting, and let $A=P_{2}-R_{2}-S_{2}$ be nonnegative and convergent double splitting. Suppose

$P_{1}^{-1} \geq P_{2}^{-1}$ and $P_{1}^{-1} S_{1} \leq P_{2}^{-1} S_{2}$,

then $\rho\left(W_{1}\right) \leq \rho\left(W_{2}\right)$.

Based on Lemma 3, we have the following results.

Theorem 3. Let $A=P_{1}-R_{1}-S_{1}=P_{2}-R_{2}-S_{2}$ be two M-double splittings. If $R_{1} \leq R_{2}, S_{1} \leq S_{2}$, then $\rho\left(W_{1}\right) \leq \rho\left(W_{2}\right)$.

Proof. For $i=1,2$, let

$\mathbb{M}_{i}=\left[\begin{array}{cc}P_{i} & S_{i} \\ 0 & I\end{array}\right], \mathbb{N}_{i}=\left[\begin{array}{cc}R_{i}+S_{i} & S_{i} \\ 0 & I\end{array}\right]$

Then

$\mathbb{A}=\mathbb{M}_{i}-\mathbb{N}_{i}$ and $W_{i}=\mathbb{M}_{i}^{-1} \mathbb{N}_{i}$.

Obviously, $\mathbb{A}$ is nonsingular whenever $A$ is nonsingular. Since $R_{1} \leq R_{2}, S_{1} \leq S_{2}$, then we have $R_{1}+S_{1} \leq$ $R_{2}+S_{2}, \bar{S}_{1} \leq S_{2}$. That is,

$\mathbb{N}_{1} \leq \mathbb{N}_{2}$.

From Lemma 3, we obtain that $\rho\left(W_{1}\right) \leq \rho\left(W_{2}\right)$.

Theorem 4. Let $A=P_{1}-R_{1}-S_{1}=P_{2}-R_{2}-S_{2}$ be two M-double splittings. If $P_{1} \leq P_{2}, S_{1} \leq S_{2}$, then $\rho\left(W_{1}\right) \leq \rho\left(W_{2}\right)$.

Proof. By simple computations, we obtain that

$R_{1}+S_{1}=P_{1}-A, R_{2}+S_{2}=P_{2}-A$.

It is not difficulty to find that $R_{1}+S_{1} \leq R_{2}+S_{2}$. Therefore,

$\mathbb{N}_{1} \leq \mathbb{N}_{2}$.

From Lemma 3, we obtain that $\rho\left(W_{1}\right) \leq \rho\left(W_{2}\right)$.

Compared with Theorem 1 and Corollary 1 [13], the condition $A^{-1} \geq 0$ in Theorems 3 and 4 is not necessary.

Theorem 5. Let $A \geq 0$, and let $A=P_{1}-R_{1}-S_{1}=$ $P_{2}-R_{2}-S_{2}$ be nonnegative splitting. If $P_{1}^{-1} \geq P_{2}^{-1}$ and $P_{1}^{-1} R_{1} \geq P_{2}^{-1} R_{2}$, then $\rho\left(W_{1}\right) \leq \rho\left(W_{2}\right)<1$ for $0<\rho\left(W_{2}\right)<1$. 
Proof. Obviously, $W_{1} \geq 0$ and $W_{2} \geq 0$. By the PerronFrobenius theorem [3], there exists a vector,

$x=\left[\begin{array}{l}x_{1} \\ x_{2}\end{array}\right] \geq 0, x \neq 0$,

such that $W_{2} x=\rho\left(W_{2}\right) x$, i.e.,

$$
\begin{aligned}
P_{2}^{-1} R_{2} x_{1}+P_{2}^{-1} S_{2} x_{2} & =\rho\left(W_{2}\right) x_{1}, \\
x_{1} & =\rho\left(W_{2}\right) x_{2} .
\end{aligned}
$$

Then we have

$$
\begin{aligned}
& W_{1} x-\rho\left(W_{2}\right) x \\
= & {\left[\begin{array}{c}
P_{1}^{-1} R_{1} x_{1}+P_{1}^{-1} S_{1} x_{2}-\rho\left(W_{2}\right) x_{1} \\
x_{1}-\rho\left(W_{2}\right) x_{2}
\end{array}\right] } \\
= & {\left[\begin{array}{c}
\left(P_{1}^{-1} R_{1}-P_{2}^{-1} R_{2}\right) x_{1}+\frac{1}{\rho\left(W_{2}\right)}\left(P_{1}^{-1} S_{1}-P_{2}^{-1} S_{2}\right) x_{1} \\
x_{1}-\rho\left(W_{2}\right) x_{2}
\end{array}\right] }
\end{aligned}
$$

Since $P_{1}^{-1} R_{1} \geq P_{2}^{-1} R_{2}$ and $0<\rho\left(W_{2}\right)<1$, then

$$
\begin{aligned}
& W_{1} x-\rho\left(W_{2}\right) x \\
\leq & \frac{1}{\rho\left(W_{2}\right)}\left[\begin{array}{c}
\left.\left(P_{1}^{-1} R_{1}-P_{2}^{-1} R_{2}\right) x_{1}+\left(P_{1}^{-1} S_{1}-P_{2}^{-1} S_{2}\right) x_{1}\right] \\
0
\end{array}\right] \\
= & \frac{1}{\rho\left(W_{2}\right)}\left[\begin{array}{c}
\left.\left(P_{1}^{-1} R_{1}+P_{1}^{-1} S_{1}\right) x_{1}-\left(P_{2}^{-1} R_{2}+P_{2}^{-1} S_{2}\right) x_{1}\right] \\
0
\end{array}\right] \\
= & \frac{1}{\rho\left(W_{2}\right)}\left[\begin{array}{c}
\left.P_{1}^{-1}\left(R_{1}+S_{1}\right) x_{1}-P_{2}^{-1}\left(R_{2}+S_{2}\right) x_{1}\right] \\
0
\end{array}\right] \\
= & \frac{1}{\rho\left(W_{2}\right)}\left[\begin{array}{c}
P_{1}^{-1}\left(P_{1}-A\right) x_{1}-P_{2}^{-1}\left(P_{2}-A\right) x_{1} \\
0
\end{array}\right] \\
= & \frac{1}{\rho\left(W_{2}\right)}\left[\begin{array}{c}
\left(P_{2}^{-1}-P_{1}^{-1}\right) A x_{1} \\
0
\end{array}\right] \leq 0 .
\end{aligned}
$$

From Lemma 1, we obtain that $\rho\left(W_{1}\right) \leq \rho\left(W_{2}\right)<1$ for $0<\rho\left(W_{2}\right)<1$.

Based on Theorem 5, we have the following result.

\section{Corollary 2. Let}

$A_{1}=P_{1}-R_{1}-S_{1}, A_{2}=P_{2}-R_{2}-S_{2}$

be nonnegative splitting. If $P_{1}^{-1} A_{1} \geq P_{2}^{-1} A_{2}$ and $P_{1}^{-1} R_{1} \geq P_{2}^{-1} R_{2}$, then $\rho\left(W_{1}\right) \leq \rho\left(W_{2}\right)<1$ for $0<\rho\left(W_{2}\right)<1$.

By investigating Corollary 2, it is easy to see that the conditioners of Corollary 2 are weaker than that of Theorem 3.1 [11]. That is, the result of Corollary 2 holds without $A_{1}^{-1} \geq 0$ and $A_{2}^{-1} \geq 0$.

Similarly, we have the following result.

Theorem 6. Let $A \geq 0$, and let $A=P_{1}-R_{1}-S_{1}=$ $P_{2}-R_{2}-S_{2}$ be nonnegative splitting. If $P_{1}^{-1} \geq P_{2}^{-1}$ and $P_{1}^{-1} S_{1} \leq P_{2}^{-1} S_{2}$, then $\rho\left(W_{1}\right) \leq \rho\left(W_{2}\right)<1$ for $0<\rho\left(W_{2}\right)<1$.

Compared with Theorem 2 [13], the condition $A^{-1} \geq 0$ in Theorem 6 is not necessary and instead of it is $A \geq 0$. By investigating Theorem 6 , it is easy to see that the conditioners of Theorem 6 are weaker than that of Theorem 2 [13].

\section{Corollary 3. Let}

$A_{1}=P_{1}-R_{1}-S_{1}, A_{2}=P_{2}-R_{2}-S_{2}$

be nonnegative splitting. If $P_{1}^{-1} A_{1} \geq P_{2}^{-1} A_{2}$ and $P_{1}^{-1} S_{1} \leq P_{2}^{-1} S_{2}$, then $\rho\left(W_{1}\right) \leq \rho\left(W_{2}\right)<1$ for $0<\rho\left(W_{2}\right)<1$.

In fact, Corollaries 2 and 3 are mainly results in [16], which implies that Theorems 5 and 6 extend the results of Corollaries 2 and 3 in [16]

\section{Numerical examples}

In this section, we make use of two examples to illustrate Theorem 3, Theorem 4 and Theorem 5.

\section{Example 4.1 Let}

$$
\begin{aligned}
& A=\left[\begin{array}{cc}
2 & -2 \\
-2 & 3
\end{array}\right], \\
& P_{1}=\left[\begin{array}{cc}
2 & 0 \\
-1 & 4
\end{array}\right], R_{1}=\left[\begin{array}{ll}
0 & 0 \\
1 & 0
\end{array}\right], S_{1}=\left[\begin{array}{ll}
0 & 2 \\
0 & 1
\end{array}\right], \\
& P_{2}=\left[\begin{array}{ll}
2 & 0 \\
0 & 5
\end{array}\right], R_{2}=\left[\begin{array}{ll}
0 & 0 \\
1 & 1
\end{array}\right], S_{2}=\left[\begin{array}{ll}
0 & 2 \\
1 & 1
\end{array}\right] .
\end{aligned}
$$

In this case, one can easily see that $R_{1} \leq R_{2}, S_{1} \leq S_{2}$, which satisfies the conditions of Theorem 3. In the meanwhile, one can also easily see that $P_{1} \leq P_{2}, S_{1} \leq S_{2}$, which satisfies the conditions of Theorem 4.

By the simple computations, we have $\rho\left(W_{1}\right)=0.8846$ and $\rho\left(W_{2}\right)=0.9164$. Clearly, $\rho\left(W_{1}\right) \leq \rho\left(W_{2}\right)<1$ hold. That is to say, Theorems 3 and 4 holds true.

\section{Example 4.2 Let}

$$
\begin{aligned}
& A=\left[\begin{array}{ll}
1 & 0 \\
0 & 2
\end{array}\right], \\
& P_{1}=\left[\begin{array}{ll}
3 & 0 \\
0 & 3
\end{array}\right], R_{1}=\left[\begin{array}{ll}
1 & 0 \\
0 & 1
\end{array}\right], S_{1}=\left[\begin{array}{ll}
1 & 0 \\
0 & 0
\end{array}\right], \\
& P_{2}=\left[\begin{array}{ll}
4 & 0 \\
0 & 5
\end{array}\right], R_{2}=\left[\begin{array}{ll}
1 & 0 \\
0 & 1
\end{array}\right], S_{2}=\left[\begin{array}{ll}
2 & 0 \\
0 & 2
\end{array}\right] .
\end{aligned}
$$

Then

$P_{1}^{-1}=\left[\begin{array}{ll}\frac{1}{3} & 0 \\ 0 & \frac{1}{3}\end{array}\right], P_{2}^{-1}=\left[\begin{array}{cc}\frac{1}{4} & 0 \\ 0 & \frac{1}{5}\end{array}\right]$.

and

$P_{1}^{-1} R_{1}=\left[\begin{array}{ll}\frac{1}{3} & 0 \\ 0 & \frac{1}{3}\end{array}\right], P_{2}^{-1} R_{2}=\left[\begin{array}{cc}\frac{1}{4} & 0 \\ 0 & \frac{1}{5}\end{array}\right]$.

That is to say, we have $P_{1}^{-1} \geq P_{2}^{-1}$ and $P_{1}^{-1} R_{1} \geq P_{2}^{-1} R_{2}$, which satisfies the conditions of Theorem 5 .

By the simple computations, we have $\rho\left(W_{1}\right)=0.7676$ and $\rho\left(W_{2}\right)=0.7696$. Clearly, $\rho\left(W_{1}\right) \leq \rho\left(W_{2}\right)<1$ holds. That is to say, Theorem 5 holds true. 


\section{Acknowledgement}

This research was supported by NSFC (No.11301009), Science \& Technology Development Plan of Henan Province (No.122300410316) and by Natural Science Foundations of Henan Province (No.13A110022). The authors thank the Editor-in-Chief M. Abdel-Aty and the anonymous referees for their constructive suggestions and helpful comments.

\section{References}

[1] Z.I. Woźnicki, Estimation of the optimum relaxation factors in partial factorization iterative methods, SIAM J. Matrix Anal. Appl., 14, 59-73 (1993).

[2] R.S. Varga, Matrix Iterative Analysis, Springer, Berlin, (2000).

[3] A. Berman, R.J. Plemons, Nonnegative Matrices in the mathematics Sciences, SIAM, Philadelphia, PA, (1994).

[4] D.M. Young, Iterative Solution of Large Linear Systems, Academic Press, New York, (1971).

[5] Z.I. Woźnicki, Nonnegative splitting theory, Japan J. Indust. Appl. Math., 11, 289-342 (1994).

[6] Z.I. Woźnicki, Basic comparison theorems for weak and weaker matrix splittings, Electron. J. Linear Algebra, 8, 5359 (2001).

[7] M. Benzi, D.B. Szyld, Existence and uniqueness of splittings for stationary iterative methods with applications to alternating methods, Numer. Math., 76, 309-321 (1997).

[8] L. Elsner, A. Frommer, R. Nabben, H. Schneider, D.B. Szyld, Conditions for strict inequality in comparisons of spectral radii of splittings of different matrices, Linear Algebra Appl, 363, 65-80 (2003).

[9] S.-Q. Shen, T.-Z. Huang, Convergence and comparison theorems for double splittings of matrices, Comput. Math. Appl., 51, 1751-1760 (2006).

[10] S.-Q. Shen, T.-Z. Huang, J.-L. Shao, Convergence and comparison results for double splittings of Hermitian positive definite matrices, Calcolo, 44, 127-135 (2007).

[11] S.-X. Miao, B. Zheng, A note on double splittings of different monotone matrices, Calcolo., 46, 261-266 (2009).

[12] C.-Y. Zhang, On convergence of double splitting methods for non-Hermitian positive semidefinite linear systems, Calcolo., 47, 103-112 (2010).

[13] J. Song, Y.-Z. Song, Convergence for nonnegative double splittings of matrices, Calcolo., 48, 245-260 (2011).

[14] L.J. Cvetković, Two-sweep iterative methods, Nonlinear Anal., 30, 25-30 (1997).

[15] W. Li, L. Elsner, L. Lu, Comparions of spectral radii and the theroem of Stein-Rosenberg, Linear Algebra Appl, 348, 283-287 (2002).

[16] C.-X. Li, Q.-F. Cui, S.-L. Wu, Comparison theorems for single and double splittings of Matrices, J. Appl. Math., 2013, Article ID 827826, 4 pages.

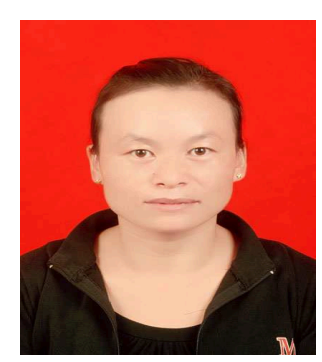

\section{Cui-Xia Li}

received her MS degree in numerical mathematics from Yunnan University. Currently, she is a lecturer in the Mathematics and Statistics Department, Anyang Normal University, Anyang, China. Her main research interests include Matrix analysis and its application, Numerical methods for saddle point problems and Numerical analysis for linear systems.

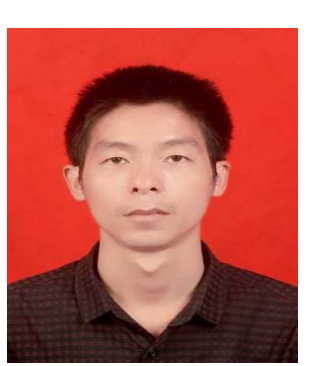

\section{Shi-Liang Wu}

has obtained his $\mathrm{PhD}$ in applied mathematics from University of Electronic Science and Technology of China. Currently, he is an associate professor in Mathematics and Statistics Department, Anyang Normal University, Anyang, China. His main research interests include Matrix analysis and its application, Numerical methods for differential-algebraic equations, Numerical partial differential equations and Numerical analysis for linear systems. 